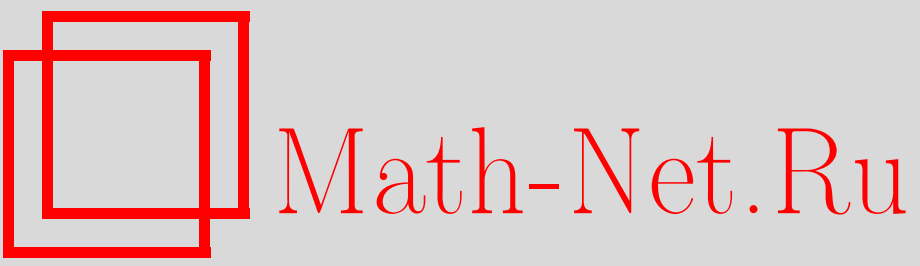

И. А. Дынников, Полином Александера многих переменных выражается через инварианты Васильева, УМН, 1997, том 52, выпуск 1, 227-228

DOI: https://doi.org/10.4213/rm810

Использование Общероссийского математического портала Math-Net.Ru подразумевает, что вы прочитали и согласны с пользовательским соглашением

http://www . mathnet.ru/rus/agreement

Параметры загрузки:

IP : 54.92 .164 .108

26 апреля 2023 г., 03:34:26 


\title{
ПОЛИНОМ АЛЕКСАНДЕРА МНОГИХ ПЕРЕМЕННЫХ ВЫРАЖАЕТСЯ ЧЕРЕЗ ИНВАРИАНТЫ ВАСИЛЬЕВА
}

\author{
И. А. Дынников
}

Придуманное Васильевым [1] определение инварианта конечного порядка для зацеплений привело к построению красивой теории, содержащей в себе почти все известные полиномиальные инварианты. Бар-Натан, Бирман и Лин [2], [3] доказали, что из равенства всех инвариантов Васильева у двух зацеплений следует, что у них также равны полиномы HOMFLY и Кауффмана, и, как частные случаи HOMFLY, полиномы Джонса, Конвея и полином Александера от одной переменной. Полином Александера от многих переменньх (описание которого с помощью локальных соотношений, данное в [4], существенно сложнее) выпал из этого списка. В настоящей заметке восполняется этот пробел.

Полином Александера от многих переменных определяется для упорядоченных ориентированных зацеплений [5]. Каждой компоненте соответствует своя переменная. Для упорядоченных зацеплений определение инварианта конечного порядка не требует модификации.

В стандартном определении полинома Александера имеется неоднозначность в выборе знака и умножении на мономы вида $t_{i}^{p}$. Это связано с тем, что полином Александера является инвариантом типа кручения (см. [6]), и создает трудности в изучении его взаимоотношений с инвариантами конечного порядка. Мы дадим ниже определение нормированного полинома Александера многих переменных, которьй однозначно определяется по диаграмме, и докажем, что все его коэффициенты после некоторой замены переменных являются инвариантами Васильева. Мы сразу будем давать определение для зацеплений в $\mathbb{R}^{3}$, которым разрешено иметь конечное число точек самопересечения. Рассматриваются зацепления с числом компонент $\geqslant 2$.

Определение нормированного полинома Александера мы начнем с простого алгебраического утверждения. Введем предварительно следующее обозначение. Для матрицы $A=\left(a_{i j}\right)_{1 \leqslant i, j \leqslant n}$ обозначим через $\widetilde{A}=\left(\widetilde{a}_{i j}\right)_{1 \leqslant i, j \leqslant n}$ матрицу, $i j$-й элемент которой равен $\widetilde{a}_{i j}=(-1)^{i+j} \Delta_{j i}(A)$, где $\Delta_{j i}(A)$ - минор, дополнительный к $a_{j i}$.

Лемма. Пусть К - коммутативное кольцо с однозначным разложсением на множители, $A$ - матрица $n \times n$ с әлементами из $\mathscr{K}, r=\left(r_{1}, \ldots, r_{n}\right)$ u $g=\left(g_{1}, \ldots, g_{n}\right)$ - две строки взаимнопростых әлементов кольца $\mathscr{K}$. Тогда если $\mathrm{r} A=0, A g^{T}=0$, то существует, и притом единственный, әлемент $\lambda=\lambda(A, r, g) \in \mathscr{K}$ такой, что $\widetilde{A}=g^{T} \lambda r$, m.e. $\widetilde{a}_{i j}=\lambda g_{i} r_{j}$.

В нашем случае кольцом $\mathscr{K}$ будет кольцо $\mathbb{Z}\left[t_{1}^{1 / 2}, t_{1}^{-1 / 2}, \ldots, t_{k}^{1 / 2}, t_{k}^{-1 / 2}\right]$ лорановских полиномов с целыми коэффициентами от $k$ переменных $t_{i}^{1 / 2}, i=1, \ldots, k$.

Пусть $D$ - диаграмма упорядоченного зацепления $L$ из $k \geqslant 2$ компонент, имеющего, возможно, простые точки самопересечения. Пусть $\gamma_{i}: S^{1} \rightarrow \mathbb{R}^{2}$ - кривая, представляющая $i$-ю компоненту зацепления $L$ на диаграмме $D$. Обозначим через $c_{i}$ степерь отображения Гаусса $S^{1} \rightarrow S^{1}$, заданного формулой $s \mapsto \dot{\gamma}_{i}(s) /\left|\dot{\gamma}_{i}(s)\right|$. Для нормировки полинома Александера нам понадобится следующий множитель: $\alpha=\prod_{i=1}^{k} t_{i}^{c_{i} / 2}$.

Для точки $x \in \mathbb{R}^{2}$, не лежащей на проекции $i$-й компоненты, обозначим через $d_{i}(x)$ степень отображения $S^{1} \rightarrow S^{1}$, определенного по формуле $s \mapsto\left(\gamma_{i}(s)-x\right) /\left|\gamma_{i}(s)-x\right|$. Для точки $x \notin D$ положим $w(x)=\prod_{i=1}^{k} t_{i}^{d_{i}(x)}$.

Разобьем кривые, составляющие диаграмму $D$, на конечное число $\partial y 2$, так чтобы никакая дуга не содержала внутри себя точки пересечения диаграммы, а начало и конец каждой дуги были различными. Пусть число дуг равно $n$. Занумеруем их произвольным образом. Для $i$-й дуги мы обозначаем номер компоненты, частью которой она является, через $(i)$. Положим $g_{i}=t_{(i)}-1$. Элементы матрицы Александера (нормированной) и строки $r$ определяются согласно правилам, указанньмм ниже.

Работа выполнена при финансовой поддержке Российского фонда фундаментальных исследований (грант № 96-01-01404). 


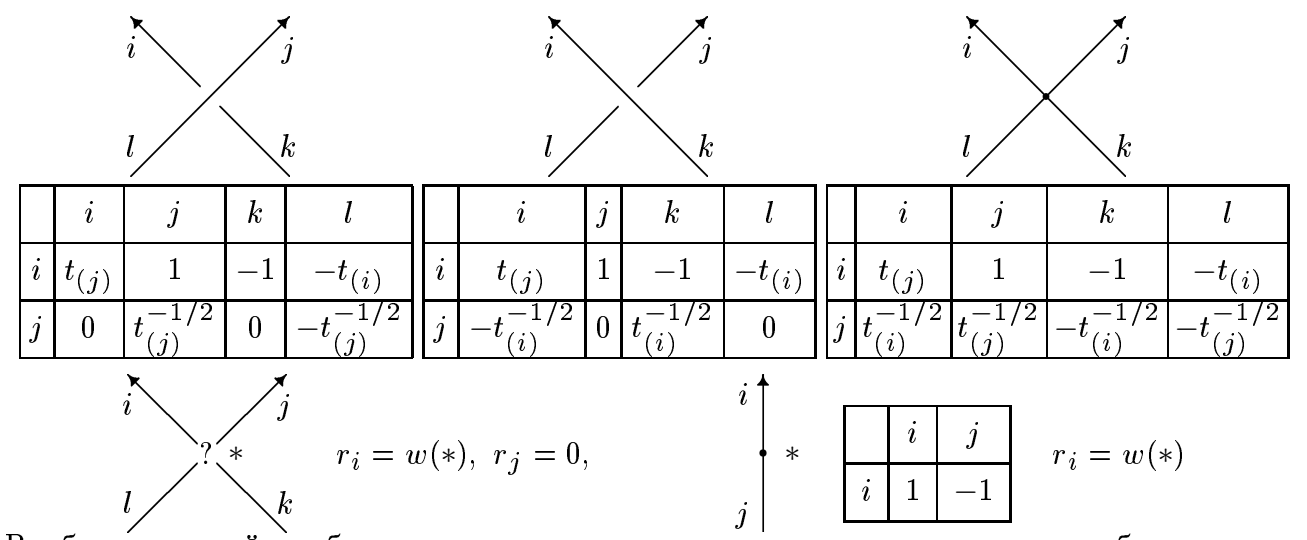

В таблицах первый столбец указывает номера строк, а первая строка - номера столбцов матрицы Александера, в которые следует записывать прочие элементы таблицы. Все оставшиеся элементы матрицы полагаются равными нулю. Знак “?”" обозначает произвольный из трех возможных типов пересечения.

Нетрудно проверить, что полученные таким образом матрица $A$ и строки $r, g$ удовлетворяют условию леммы. Определим нормированньй полином Александера по формуле $\Delta_{L}=\alpha \lambda(A, r, g) \in \mathscr{K}$. Классический полиномом Александера есть $\Delta_{L}$ с точностью до умножения на обратимый элемент колца $\mathscr{K}$. Следующее утверждение можно получить простым вычислением с нормированной матрицей Александера.

Теорема 1. 1) Данное выше определение полинома $\Delta_{L}$ корректно, т.е. не зависит от выбора диаграммы, изображающей зачепление $L$, произвола в разбиении этой диаграммы на дуги и их нумерачии.

2) Нормированный полином Александера удовлетворяет локальному соотношению Васильева:

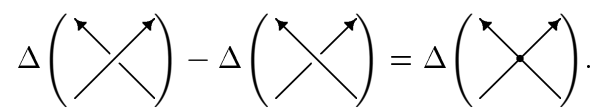

Сделаем теперь в полиноме Александера замену переменных: $t_{i}=e^{x_{i}}$. Получим ряд $\widehat{\Delta}_{L} \in$ $\mathbb{Z}\left[\left[x_{1}, \ldots, x_{k}\right]\right]$.

Теорема 2. Если зацепление L имеет $m$ точек самопересечения, то ряд $\widehat{\Delta}_{L}$ не содержит ненулевых членов степени $<m-1$.

ДоКАЗАТЕЛЬСтво. Вспомним определение полинома Александера. Если $r_{i} \neq 0$, то $\Delta_{L}=$ $\alpha(-1)^{i+j} \frac{\Delta_{i j}(A)}{r_{i} g_{j}}$. Каждому самопересечению зацепления $L$ соответствует пара строк в матрице Александера, совпадающих в нулевом члене после указанной замены переменных. Отсюда миноры $\Delta_{i j}(A)$, выраженные через переменные $x_{i}$, имеют порядок $O\left(|x|^{m}\right)$. Элементы $\alpha$ и $r_{i}$ кольца $\mathscr{K}$, а значит, и их образы в $\mathbb{Z}\left[\left[x_{1}, \ldots, x_{k}\right]\right]$, обратимы. Элемент $g_{i}$ имеет вид $g_{i}=t_{i}-1=$ $x_{i}+\cdots$.

Таким образом, коэффициенты ряда $\widehat{\Delta}_{L}$ при членах степени $m$ являются инвариантами Васильева порядка $\leqslant m+1$. Отметим, что аналогичная конструкция для полиномов HOMFLY и Кауффмана дает инвариант порядка $m$.

\section{СПИСОК ЛИТЕРАТУРЫ}

[1] Vassiliev V. A. Cohomology of knot spaces // Theory of singularities and its Applications / ed. V.I. Arnold. Providence: Amer. Math. Soc., 1990. [2] Bar-Natan D. // Topology. 1995. V. 34. № 2. P. 423-472. [3] Birman J. S., Lin X-S. Knot polynomials and Vassiliev's invariants // Preprint: Colombia Univ., 1991. [4] Murakami J. // Pacific J. Math. 1993. V. 157. № 1. P. 109-135. [5] Torres G. // Ann. of Math. 1953. V. 57. № 1. P. 57-89. [6] Тураев В. Г. // УМН. 1986. T. 41. № 1. С. 97-147. 